Mhicrowave Engineering

\title{
Accuracy Evaluation for Antenna Measurements at mm-Wave Frequencies
}

Linus Boehm, Frank Boegelsack, Martin Hitzler, Stefan Wiehler, and Christian Waldschmidt

(C) 2016 IEEE. Personal use of this material is permitted. Permission from IEEE must be obtained for all other uses, in any current or future media, including reprinting/republishing this material for advertising or promotional purposes, creating new collective works, for resale or redistribution to servers or lists, or reuse of any copyrighted component of this work in other works.

DOI: $10.1109 /$ EuCAP.2016.7481972 


\title{
Accuracy Evaluation for Antenna Measurements at mm-Wave Frequencies
}

\author{
Linus Boehm, Frank Boegelsack, Martin Hitzler, Stefan Wiehler, and Christian Waldschmidt \\ Institute of Microwave Engineering \\ University of Ulm \\ Ulm, Germany \\ forename.surname@uni-ulm.de
}

\begin{abstract}
Measurements of integrated antennas are often cumbersome and erroneous. Inaccuracies of the measurement setup, unknown material parameters, and metal parts in the immediate surrounding of the antenna under test (AUT) cause errors to the measured parameters. In order to be able to measure integrated antennas at frequencies beyond $100 \mathrm{GHz}$ with high accuracy, a dedicated measurement setup was developed. For the quantification of the accuracy of the setup various measurements with different parameters were taken and their individual impact on the result was analyzed. On this basis the accuracy of measured antenna parameters like gain, directivity, and radiation pattern at $280 \mathrm{GHz}$ was calculated as well as their sensitivity towards certain uncertainties in the measurement process.
\end{abstract}

\section{INTRODUCTION}

The requirement for higher data rates and cheaper production costs drive the development of radar sensors and communication devices to frequencies beyond $100 \mathrm{GHz}$. In order to avoid bonding to external antennas, which would make the matching and the usage of the chips more difficult, the antennas are often realized on the chip itself. On-chip antennas facilitate the system design for the end user and are cost efficient as fewer external parts are required. Accurate measurement methods are needed to optimize the performance of the antenna and the entire system. However, measurements of these antennas are difficult due to the short wavelengths of the signals and interference with the measurement setup itself.

At frequencies beyond $100 \mathrm{GHz}$ the wavelengths are smaller than $3 \mathrm{~mm}$, which makes it difficult to obtain repeatable and meaningful results. Both small deviations of the measurement positions and an inaccurate alignment of the antenna under test with the reference antenna influence the measured data and lead to erroneous measured antenna parameters.

The second error source is the measurement setup itself. Integrated antennas have to be contacted with wafer probes that feed the signal directly on the chip. Probes are quite fragile and prone to be damaged by movement or vibrations. Therefore, probe and AUT need to be as stable as possible and, unlike usually, the AUT is not turned to measure the radiation pattern, but the reference antenna is moved around the AUT during a measurement. Different approaches to ensure movements as accurate as possible have been investigated [1]. The characterized setup uses a robotic arm that moves the reference antenna around the AUT to ensure a position accuracy as high

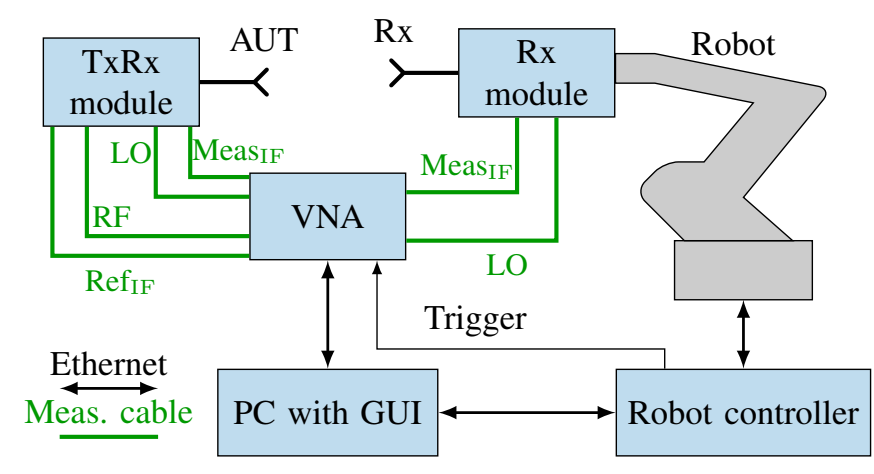

Fig. 1. Schematic of the measurement setup.

as possible. The flexibility of the robot also allows to precisely change measurement settings to evaluate the effect of certain error parameters on the result and on the overall measurement uncertainty. [2] shows a similar approach at $5 \mathrm{GHz}$.

This paper mainly focuses on the measurement setup presented in [3], but can be applied to different setups as well. A block diagram of the setup is shown in Fig. 1. The Rx antenna and the receiver module are mounted to the robotic arm, which moves the Rx antenna on a desired path around the AUT and triggers the network analyzer at the specified measurement positions.

\section{ERROR SOURCES}

Antenna measurements are affected by various error sources. A list of the identified error sources can be found in the second column of Table I. The third column specifies whether the source affects the radiation pattern (RP), the directivity (D), or the gain in direction of maximum radiation (G).

Uncertainties of the AUT position and orientation affect all three measured quantities. The phase center uncertainty of the standard gain horn (SGH), which was used for the radiation pattern, is $5 \mathrm{~mm}$ and $1 \mathrm{~mm}$ for the integrated antenna (\#4) that was used as an AUT during the gain measurements. Uncertainties of the Rx antenna (\#7-10), on the other hand, do not have an influence on the result as they disappear after normalization (RP, D), or after calibration $(G)$. 
TABLE I

UNCERTAINTY BUDGETS

\begin{tabular}{|c|c|c|c|c|c|c|c|c|c|}
\hline \multirow{2}{*}{ \# } & \multirow{2}{*}{$\begin{array}{l}\text { Source of Uncertainty } \\
x_{i}\end{array}$} & \multirow{2}{*}{$\begin{array}{l}\text { Affected } \\
\text { Measurements }\end{array}$} & \multirow{2}{*}{$\begin{array}{c}\text { Uncertainty }( \pm) \\
u\left(x_{i}\right)\end{array}$} & \multicolumn{2}{|c|}{ Radiation Pattern } & \multicolumn{2}{|c|}{ Directivity } & \multicolumn{2}{|c|}{ Gain } \\
\hline & & & & $c_{i}$ & $s_{x_{i}}$ & $c_{i}$ & $s_{x_{i}}$ & $c_{i}$ & $s_{x_{i}}$ \\
\hline 1 & AUT pos $\mathrm{x}$ & $\mathrm{RP} / \mathrm{D} / \mathrm{G}$ & $1.5 \mathrm{~mm}$ & $0.024 \mathrm{~dB} / \mathrm{mm}$ & $0.5 \mathrm{~mm}$ & $0.004 \mathrm{~dB} / \mathrm{mm}$ & $0.5 \mathrm{~mm}$ & $0.006 \mathrm{~dB} / \mathrm{mm}$ & $0.5 \mathrm{~mm}$ \\
\hline 2 & AUT pos y & $\mathrm{RP} / \mathrm{D} / \mathrm{G}$ & $1.5 \mathrm{~mm}$ & $0.248 \mathrm{~dB} / \mathrm{mm}$ & $0.5 \mathrm{~mm}$ & $0.008 \mathrm{~dB} / \mathrm{mm}$ & $0.5 \mathrm{~mm}$ & $0.041 \mathrm{~dB} / \mathrm{mm}$ & $0.5 \mathrm{~mm}$ \\
\hline 3 & AUT pos z & $\mathrm{RP} / \mathrm{D} / \mathrm{G}$ & $2 \mathrm{~mm}$ & $0.041 \mathrm{~dB} / \mathrm{mm}$ & $0.67 \mathrm{~mm}$ & $0.003 \mathrm{~dB} / \mathrm{mm}$ & $0.67 \mathrm{~mm}$ & $0.028 \mathrm{~dB} / \mathrm{mm}$ & $0.67 \mathrm{~mm}$ \\
\hline 4 & AUT phase center & $\mathrm{RP} / \mathrm{D} / \mathrm{G}$ & $5 \mathrm{~mm} / 1 \mathrm{~mm}$ & $0.023 \mathrm{~dB} / \mathrm{mm}$ & $1.67 \mathrm{~mm}$ & $0.014 \mathrm{~dB} / \mathrm{mm}$ & $1.67 \mathrm{~mm}$ & - & $\approx 0$ \\
\hline 5 & AUT tilt & $\mathrm{RP} / \mathrm{D} / \mathrm{G}$ & $0.125^{\circ}$ & $1.369 \mathrm{~dB} /{ }^{\circ}$ & $0.04^{\circ}$ & $0.06 \mathrm{~dB} /{ }^{\circ}$ & $0.04^{\circ}$ & $0.165 \mathrm{~dB} /{ }^{\circ}$ & $\approx 0$ \\
\hline 6 & AUT mismatch & G & & - & - & - & - & $\approx 0$ & - \\
\hline 7 & $\mathrm{Rx}$ tilt & - & $0.1^{\circ}$ & - & - & - & - & - & - \\
\hline 8 & Rx length & - & $100 \mu \mathrm{m}$ & - & - & - & - & - & - \\
\hline 9 & $\mathrm{Rx}$ phase center & - & $5 \mathrm{~mm}$ & - & - & - & - & - & - \\
\hline 10 & Rx Gain & - & $0.2 \mathrm{~dB}$ & - & - & - & - & - & - \\
\hline 11 & REF gain & G & $0.1 \mathrm{~dB}$ & - & - & - & - & 1 & $0.033 \mathrm{~dB}$ \\
\hline 12 & REF tilt & G & $0.125^{\circ}$ & - & - & - & - & $1.369 \mathrm{~dB} /{ }^{\circ}$ & $0.04^{\circ}$ \\
\hline 13 & REF phase center & G & $5 \mathrm{~mm}$ & - & - & - & - & $0.012 \mathrm{~dB} / \mathrm{mm}$ & $1.67 \mathrm{~mm}$ \\
\hline 14 & REF mismatch & G & & - & - & - & - & $\approx 0$ & - \\
\hline 15 & Connectors & G & $0.4 \mathrm{~dB}$ & - & - & - & - & $1 \mathrm{~dB} / \mathrm{dB}$ & $0.133 \mathrm{~dB}$ \\
\hline 16 & Trigger position & $\mathrm{RP}$ & $350 \mu \mathrm{m}$ & & & & & - & - \\
\hline 17 & Cable flex & $\mathrm{RP}$ & & $1 \mathrm{~dB} / \mathrm{dB}$ & $0.038 \mathrm{~dB}$ & $1 \mathrm{~dB} / \mathrm{dB}$ & $0.012 \mathrm{~dB}$ & - & - \\
\hline 18 & Noise & $\mathrm{RP} / \mathrm{G}$ & & & & & & $1 \mathrm{~dB} / \mathrm{dB}$ & $0.084 \mathrm{~dB}$ \\
\hline 19 & AUT-Rx-Polarization & $\mathrm{RP} / \mathrm{D} / \mathrm{G}$ & $0.1^{\circ}$ & - & $\approx 0$ & - & $\approx 0$ & - & $\approx 0$ \\
\hline 20 & Distance & & $350 \mu \mathrm{m}$ & - & - & - & - & $\approx 0$ & $\approx 0$ \\
\hline 21 & Multiple reflections & $\mathrm{RP} / \mathrm{D} / \mathrm{G}$ & & $1 \mathrm{~dB} / \mathrm{dB}$ & $0.049 \mathrm{~dB}$ & $1 \mathrm{~dB} / \mathrm{dB}$ & $0.036 \mathrm{~dB}$ & $1 \mathrm{~dB} / \mathrm{dB}$ & $0.833 \mathrm{~dB}$ \\
\hline 22 & $\phi$ resolution $\left(3^{\circ}\right)$ & D & & - & - & $1 \mathrm{dP} / \mathrm{dP}$ & $0005 \mathrm{dP}$ & - & - \\
\hline 23 & $\theta$ resolution $\left(0.25^{\circ}\right)$ & RP/D & & $\approx 0$ & - & $1 \mathrm{~dB} / \mathrm{dB}$ & $0.005 \mathrm{~dB}$ & - & - \\
\hline 24 & $\operatorname{Max} \theta$ & $\mathrm{D}$ & & - & - & $1 \mathrm{~dB} / \mathrm{dB}$ & $0.009 \mathrm{~dB}$ & - & - \\
\hline 25 & Amplitude drift (1h) & $\mathrm{D} / \mathrm{G}$ & $0.025 \mathrm{~dB}$ & - & - & - & $\approx 0$ & - & $\approx 0$ \\
\hline 26 & VNA crosstalk & RP/D & $112 \mathrm{nV}_{\mathrm{rms}}$ & - & $\approx 0$ & - & $\approx 0$ & - & $\approx 0$ \\
\hline 27 & Receiver non-linearity & $\mathrm{RP} / \mathrm{D} / \mathrm{G}$ & & $1 \mathrm{~dB} / \mathrm{dB}$ & $0.049 \mathrm{~dB}$ & $1 \mathrm{~dB} / \mathrm{dB}$ & $0.09 \mathrm{~dB}$ & - & $\approx 0$ \\
\hline 28 & Absorber placement & $\mathrm{RP} / \mathrm{D} / \mathrm{G}$ & & - & $\approx 0$ & - & $\approx 0$ & - & $\approx 0$ \\
\hline Exp & aded uncertainty $U_{c}(y)$ & $\%$ confidence) & & 0.325 & & 0.203 & & 1.70 & \\
\hline
\end{tabular}

The gain was measured using a gain comparison method, where the received signal of the AUT is compared to a reference antenna (REF) of known gain. This reference antenna, its orientation as well as any connectors that differentiate from the reference measurement (e.g. wafer probe) were analyzed for the gain measurement (\#11-15).

The difference between multiple measurements with the same parameters were attributed to the trigger position uncertainty, cable movements, and noise. Therefore, their combined contribution is shown in Table I instead of the individual contributions (\#16-18). The trigger position accuracy depends on the speed and the acceleration of the robot. For this evaluation the velocity was chosen such that the measurement time for a single plane is $1 \mathrm{~min}(-90 \leq \theta \leq 90)$ and $60 \mathrm{~min}$ for a 3D measurement $(-60 \leq \theta \leq 60,-90 \leq \phi<90)$. The IF bandwidth was set to $100 \mathrm{~Hz}$. To minimize deviations through cable movements, cable routing that ensures a defined cable position and large bending radii was used.

As all the measurements were performed in the far field of the AUT, no near field to far field conversion was necessary. Phase uncertainties, which are caused by cables that flex due to the robot movement [4] and [5], were therefore not investigated.

Rx antenna and AUT were aligned after performing a polarization scan of the AUT. The influence of the AUT-Rxpolarization uncertainty (\#19) is therefore negligible.

As long as the measurement is taken in the far field of the AUT, the distance does have an insignificant effect on the radiation pattern or the directivity. During a measurement the distance (\#20) solely depends on the robot accuracy. The uncertainty is way smaller than the uncertainty of the AUT position and thus negligible.

In order to be able to measure integrated antennas, the setup contains a probe station. Its metal surface causes reflections and scattering, which impairs the measurements. The influence of reflections (\#21) was evaluated by comparing measurements with a distance difference of $\Delta d=\frac{\lambda}{4}$. To minimize reflections, the setup is in an anechoic box and both robot and probe station are covered with absorbers. As the test range was intended for relatively small antennas, far field measurements can be performed without additional reflectors that would affect amplitude and phase and reduce the size of the quiet zone [6]. Therefore, the size of the quiet zone primarily depends on reflections that occur despite the used absorbers. Because of the high attenuation at mm-wave frequencies, no dedicated quiet zone analysis was performed.

Other uncertainties were found to have a negligible impact and were not taken into account in the final uncertainty 
analysis. Among these are AUT and SGH mismatch, amplitude drift and crosstalk (\#25, 26).

For the radiation pattern and the directivity uncertainty analysis a horn antenna was used. For the gain analysis an integrated antenna was measured with a standard gain horn as a reference. All measurements were performed at $280 \mathrm{GHz}$.

\section{UNCERTAINTY BUDGETS}

In order to derive the uncertainty budget for directivity, gain, and radiation pattern measurements, the different error sources $x_{i}$ were evaluated and their individual impact on the respective measured quantity $y$ (e.g. gain or directivity) was quantified as shown in [7]. Knowing the impact of a particular error source $x_{k}$ on $y$, the sensitivity of $y$ towards $x_{k}$ can be calculated with

$$
c_{k}=\frac{\mathrm{d} y\left(x_{k}\right)}{\mathrm{d} x_{k}} \text {. }
$$

The sensitivities were either found through propagation of error considerations or through repeated measurements.

If the relation between the parameters $x_{k}$ and $y$ was known, propagation of error was used. With propagation of error the sensitivity is determined by calculating the influence of $x_{k}$ on the final result $y$. This was used for example to calculate the sensitivity of the gain towards a false measurement distance, as the relation between distance and received amplitude, and thus the measured gain can be calculated easily with the free space attenuation (see Section VI).

When the effect of the uncertainty of the measurement parameter was not known, the sensitivity was quantified through multiple measurements, in which the error source $x_{k}$ was deliberately altered in order to introduce an error. By comparing the erroneous measurement result $y_{\text {err }}$ with a reference measurement $y_{\text {ref }}$, where no deliberate error was introduced, the effect of this specific measurement uncertainty can be determined and the sensitivity can then be calculated.

If the sensitivity of the result towards every source of error is known, the overall uncertainty budget $u_{c}(y)$ can be calculated with

$$
u_{c}(y)=\sqrt{\sum_{i=1}^{N}\left(c_{i} \cdot s_{x_{i}}\right)^{2}},
$$

where $N$ is the total number of uncertainties taken into account. $s_{x_{i}}$ is the standard deviation of a certain $x_{i}$. It is obtained by multiplying the maximum deviation $u\left(x_{i}\right)$ with a factor depending on the distribution of $x_{i}$. For normal distributed errors, which was assumed for all parameters for this analysis, the factor is $\frac{1}{3}$ [7]. In order to obtain a higher level of confidence, the extended uncertainty $U_{c}(y)=k \cdot u_{c}(y)$ can be calculated. In this paper $k=2$ was used, which corresponds to a $95 \%$ confidence [7].

\section{RAdiation PATtern UnCERTAinty Budget}

For the radiation pattern uncertainty budget the H-plane of a standard gain horn was used. A comparison between measurement and simulation of the antenna is shown in Fig. 2 The deviations between the plots are caused by production

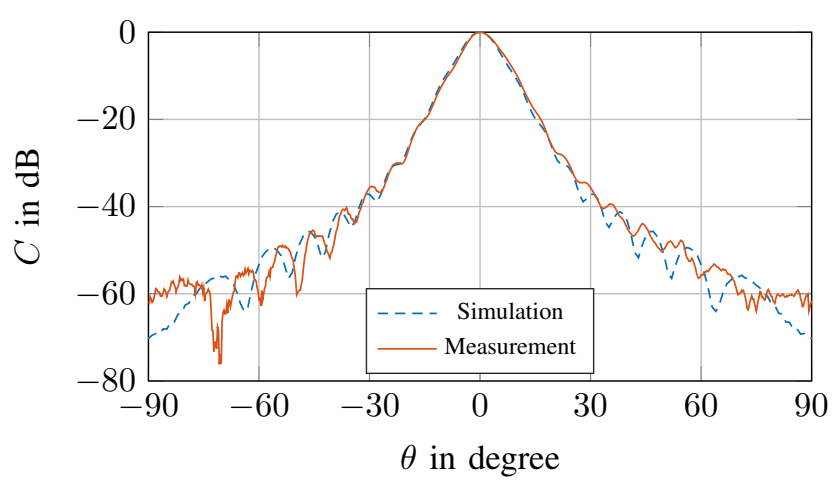

Fig. 2. Measurement of a horn antenna in the H-plane horn at $280 \mathrm{GHz}$.

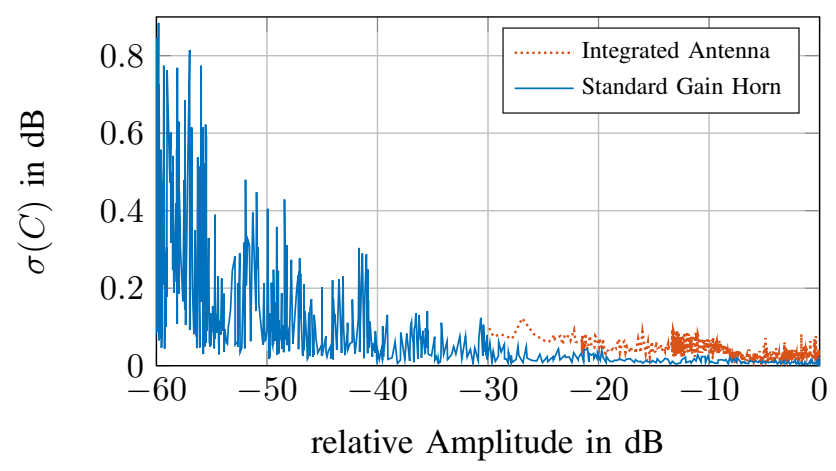

Fig. 3. Standard deviation of 10 radiation pattern measurements at $280 \mathrm{GHz}$.

inaccuracies of the horn, noise, and multiple reflections that occur despite the usage of absorbers.

The setup was designed for integrated antennas, which usually do not have a high gain. Therefore, the uncertainty budget was calculated within $30 \mathrm{~dB}$ of the main lobe to cover the dynamic range of an integrated antenna in a half sphere. For a horn antenna the standard deviation between 10 measurements increases significantly for lower relative amplitudes due to multiple reflections as shown in Fig. 3. The standard deviation of an integrated antenna remains relatively stable over the entire dynamic range.

The radiation pattern results contain random deviations caused by cable movements, noise, and trigger position inaccuracies. When a measurement parameter $x_{k}$ is changed to analyze the effect on the radiation pattern, the contribution of the random errors must be minimized. This was achieved by averaging over the radiation patterns $C(\theta)$ of 10 measurements for both the reference measurement and an erroneous measurement to obtain the averaged radiation pattern $\bar{C}(\theta)$.

In Fig. 4 the absolute difference

$$
C_{\text {diff, abs }}(\theta)=\left|\bar{C}_{x}(\theta)-\bar{C}_{\text {ref }}(\theta)\right|
$$

between a reference measurement $\bar{C}_{\text {ref }}$ and a second averaged reference measurement $\bar{C}_{\text {ref }}$ (blue) is shown. The mean value of the difference for the reference measurements is $\bar{C}_{\text {diff, abs }}=0.022 \mathrm{~dB}$. For a measurement with erroneous 


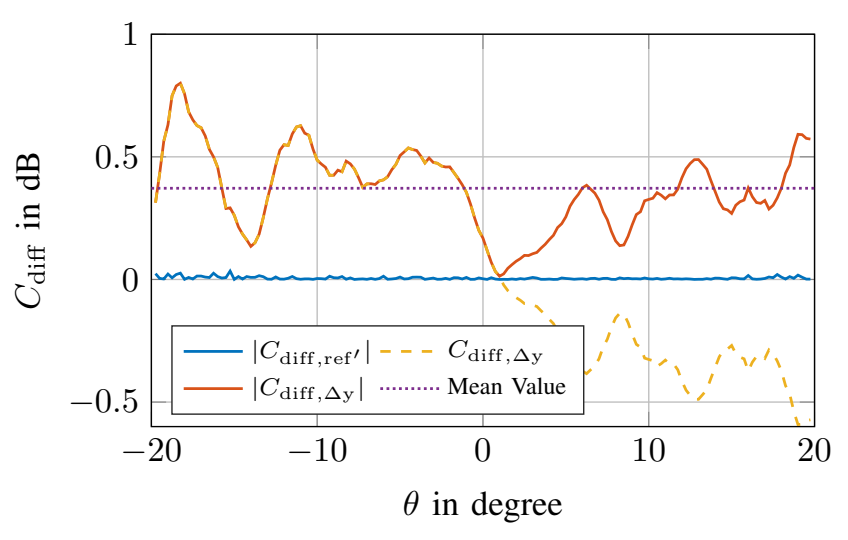

Fig. 4. Absolute difference between $\bar{C}_{\text {ref }}$ \& $\bar{C}_{\text {ref }}$ and $\bar{C}_{\Delta \mathrm{y}} \& \bar{C}_{\text {ref }}$.

$y$-position (red) of the AUT, the mean value of $C_{\text {diff,abs }}$ is $\bar{C}_{\text {diff, abs }}=0.372 \mathrm{~dB}$. As the mean difference increased by a factor of more than 10 , this deviation can be fully attributed to $\Delta y_{\text {pos }}$ and the noise influence can be neglected. The sensitivity towards the $y$-position (assuming linear dependencies) is:

$$
c_{y-\mathrm{pos}}=\frac{\bar{C}_{\mathrm{diff}, \mathrm{abs}}}{\Delta y_{\mathrm{pos}}}=\frac{0.372 \mathrm{~dB}}{1.5 \mathrm{~mm}}=0.248 \frac{\mathrm{dB}}{\mathrm{mm}} .
$$

The measurement was taken on a semicircle in the $y$-zplane. As the AUT position was not in the center of the circle, the results are too high for negative $\theta$ values and too low for positive $\theta$ values as shown in Fig. 4 (yellow curve).

The extended uncertainty budget for the horn antenna measurement is $0.325 \mathrm{~dB}$ (95\% confidence). The uncertainties do not only depend on the setup itself, but also on the AUT that is measured. Because of differences in feeding and different patterns the sensitivities and the overall uncertainty budget can change.

Integrated antennas have to be fed with wafer probes, which increase reflections and alter the radiated field depending on the probe placement on the chip. Due to the lower gain, the received signal level is lower, which leads to a higher noise contribution and an increased standard deviation in Fig. 3 compared to the horn antenna. On the other hand the slope of the pattern decreases significantly resulting in a lower sensitivity towards AUT tilt and $x$ - $y$-AUT position deviations.

\section{Directivity UnCERTAinty Budget}

The directivity can be calculated by integrating over the radiation pattern $C(\theta, \phi)$ on a sphere around the AUT:

$$
D_{i}=\frac{4 \pi}{\int_{\phi=0}^{\pi} \int_{\theta=-\pi}^{\pi} C^{2}(\theta, \phi) \sin (\theta) \mathrm{d} \theta \mathrm{d} \phi} .
$$

This integration over many measurement points averages over the noise, which is why only one measurement was taken for each variation of $x_{i}$ for directivity measurements. The smaller noise contribution can be seen in Table I (\#16-18). The combined standard deviation of trigger position, cable flex, and noise is reduced by $2 / 3$ to $0.012 \mathrm{~dB}$, compared to

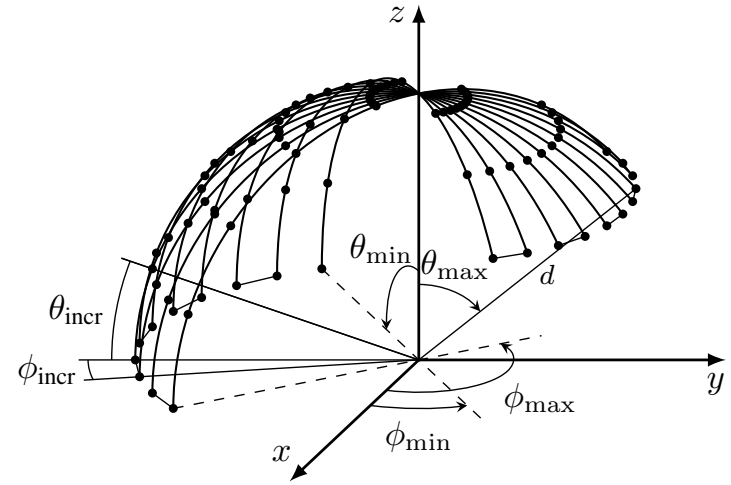

Fig. 5. Schematic of the measurement setup.

the uncertainty of the radiation pattern measurement, where it was $0.038 \mathrm{~dB}$. As the measurement integrates over a 2D field around the AUT, false AUT positions also have a significantly smaller impact on the result $(\# 1-5)$, leading to a lower expanded uncertainty budget of $0.203 \mathrm{~dB}$ for directivity measurements of horn antennas. It was observerd that errors that occur due to the discrete integration over the field $(\# 22,23)$ only have a minor effect on the uncertainty budget.

In order to calculate the directivity of the AUT, the radiated field must be integrated on a full sphere around the AUT However, the movement of the robot is limited due to both the maximum angles of the robot joints and the probe station, which does not allow the robot to measure in the lower hemisphere of the AUT (\#24). Therefore, only part of the sphere $\left(\theta_{\text {min }}<\theta_{\text {meas }}<\theta_{\text {max }}\right.$, see Fig. 5) can be measured and integrated over, which causes the integral to be too small.

For a lower bound, the part of the field that cannot be measured can be assumed to be zero, i.e. $C\left(|\theta|>\theta_{\max }, \phi\right)=0$. As the radiation decreases away from the main lobe, an upper bound would be to assume the field to be equal to the edge of the measured area, $C\left(|\theta|>\theta_{\max }, \phi\right)=C\left(|\theta|=\theta_{\max }, \phi\right)$ and $C\left(|\theta|<-\theta_{\max }, \phi\right)=C\left(|\theta|=-\theta_{\max }, \phi\right)$.

The maximum scanning angle of $\theta$ for a $3 \mathrm{D}$ scan with a radius of $250 \mathrm{~mm}$ is $-\theta_{\min }=\theta_{\max }=60^{\circ}$, which results in a lower bound for the directivity $D_{\text {lowB }}=26.24 \mathrm{dBi}$ and an upper bound of $D_{\mathrm{upB}}=26.29 \mathrm{dBi}$. The mean value is $D_{\text {mean }}=26.27 \mathrm{dBi}$ and agrees with the simulated value of $26.15 \mathrm{dBi}$. The sensitivity towards $\theta_{\max }$ is therefore $c_{\theta_{\max }}=$ $\frac{1}{3}\left(D_{\text {mean }}-D_{\text {lowB }}\right)=0.009 \frac{\mathrm{dB}}{\mathrm{dB}}$. As the horn antenna has a high gain and radiates most of the power in the direction that was measured, the sensitivity towards $\theta_{\max }$ is low.

\section{Gain Uncertainty Budget}

The gain was calculated using the gain comparison method, where the received power of the AUT is compared to a reference antenna of known gain. If different connectors are required to connect the two antennas, their attenuation has to be taken into account. The gain can then be calculated with

$$
\left.G_{\mathrm{AUT}}\right|_{\mathrm{dBi}}=\left.S_{21, \mathrm{AUT}}\right|_{\mathrm{dB}}-\left.S_{21, \mathrm{ref}}\right|_{\mathrm{dB}}+\left.G_{\mathrm{ref}}\right|_{\mathrm{dBi}}-\left.S_{21, \mathrm{con}}\right|_{\mathrm{dB}} .
$$




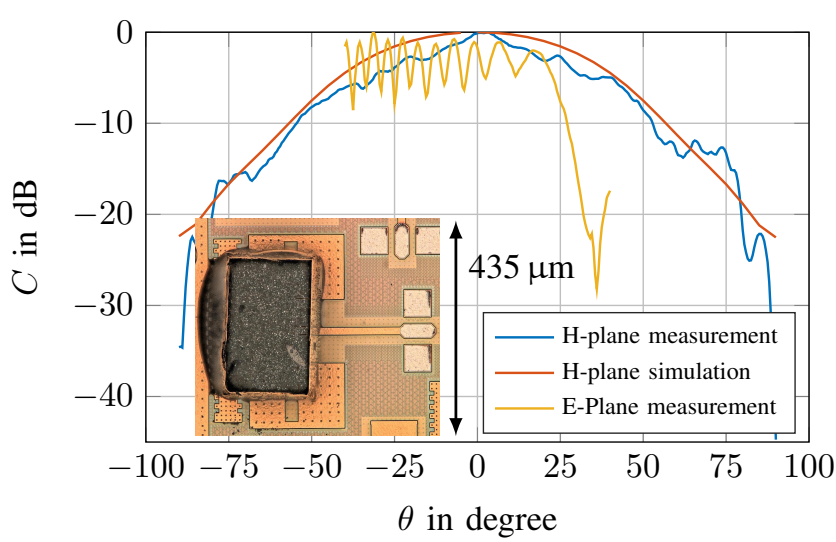

Fig. 6. Radiation pattern of an integrated antenna at $280 \mathrm{GHz}$.

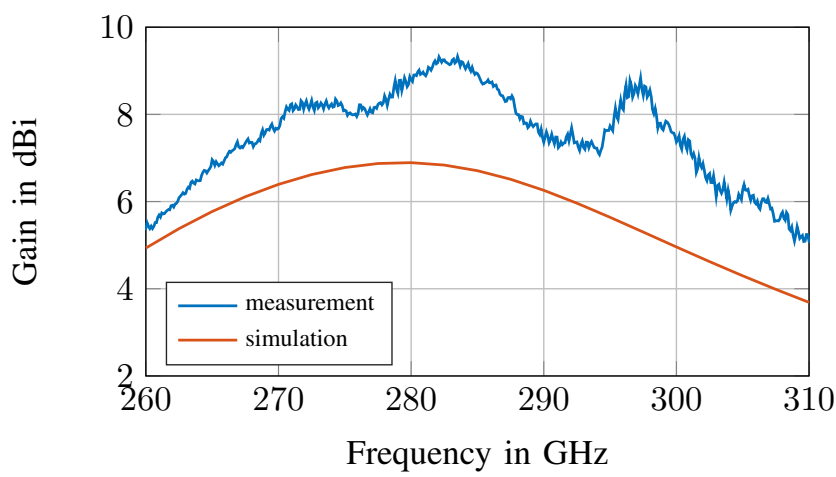

Fig. 7. Gain of the integrated antenna.

The integrated antenna shown in Fig. 6 was used as AUT. The AUT was contacted with a wafer probe. The pads and microstrip line on the chip add further attenuation to the measurement of the integrated antenna. For the reference horn measurement a waveguide bent (WGB) was used. Hence, $S_{21,\left.\mathrm{con}\right|_{\mathrm{dB}}}$ consists of three terms:

$$
\left.S_{21, \mathrm{con}}\right|_{\mathrm{dB}}=\left.S_{21, \text { probe }}\right|_{\mathrm{dB}}+\left.S_{21, \mathrm{chip}}\right|_{\mathrm{dB}}-\left.S_{21, \mathrm{WGB}}\right|_{\mathrm{dB}} .
$$

In the simulation the location of the phase center of the reference antenna changed over frequency and viewing angle with an uncertainty of $5 \mathrm{~mm}$. Using the free space attenuation formula the effect on the gain measurement can be calculated with

$$
\Delta G_{\mathrm{AUT}}=10 \log _{10}\left(\frac{\mathrm{R}+u(\mathrm{R})}{\mathrm{R}}\right) \mathrm{dB} .
$$

With a phase center distance $\mathrm{R}=350 \mathrm{~mm}$ and an uncertainty $u(\mathrm{R})=5 \mathrm{~mm}$ the error is $\Delta G_{\mathrm{AUT}}=0.062 \mathrm{~dB}$. The resulting sensitivity is $c_{\text {phase_cen_REF }}=\frac{0.062 \mathrm{~dB}}{5 \mathrm{~mm}}=0.012 \frac{\mathrm{dB}}{\mathrm{mm}}$.

The used wafer probe is very close to the AUT and reflects part of the field causing the ripples in the E-plane shown in Fig. 6. The location of the minima and maxima is very sensitive to the probe position. The amplitude of the ripples cause a $S_{21, \text { AUT }}$ uncertainty of $u$ (mult reflections $)=2.5 \mathrm{~dB}$. This is especially critical as the uncertainty of $S_{21 \text {,con adds }}$ linearly to the uncertainty of the gain measurement and, therefore, it has a strong influence on the gain error budget. The measured pattern has a maximum at $\theta \approx 0^{\circ}$, which results in an over estimation of the measured gain (see Fig. 7).

With $S_{21 \text {,probe }}=-3 \mathrm{~dB}$, which was measured by the manufacturer, the measurement result for the gain of the integrated antenna at $280 \mathrm{GHz}$ was $G_{\mathrm{AUT}}=8.76 \mathrm{dBi}$. The measured radiation pattern shown in Fig. 6 has a smaller beamwidth than the simulated antenna, which suggests a higher gain of the antenna than in the simulation. Further reasons for the deviation between measurement and simulation can be wrong material parameters and overestimated losses in the simulation.

\section{CONCLUSION}

In the analysis of the measurement setup the uncertainty budgets for radiation pattern, directivity, and gain measurement were calculated in order to quantify the maximum deviations of the measurements taken with the setup. Mechanical, electrical, and processing influences were taken into account and their individual impact on the respective measurement result was analyzed. The expanded uncertainty budget, with a $95 \%$ confidence, is well below $0.4 \mathrm{~dB}$ for both the radiation pattern and directivity measurements of horn antennas, which benefit from the normalization that cancels out attenuation related error sources.

The uncertainty for the gain measurement of an integrated antenna is $\approx 1.7 \mathrm{~dB}$. By far the biggest source of uncertainty are caused by reflections of the wafer probe, which is required for the waveguide-to-chip transition and caused an over estimation of the gain. Deviations between simulation and measurement of up to $3 \mathrm{~dB}$ are not necessarily due to measurement errors, but can also be caused by fabrication tolerances and false material parameters in the simulation.

\section{REFERENCES}

[1] D. Titz, F. Ferrero, and C. Luxey, "Development of a Millimeter-Wave Measurement Setup and Dedicated Techniques to Characterize the Matching and Radiation Performance of Probe-Fed Antennas [Measurements Corner]," Antennas and Propagation Magazine, IEEE, vol. 54, pp. 188203, Aug 2012.

[2] S. Pivnenko, J. M. Nielsen, and O. Breinbjerg, "Very High-Accuracy Calibration of Radiation Pattern and Gain of a Near-Field Probe," in Antennas and Propagation (EuCAP), 8th European Conference on, The Hague, Netherlands, pp. 1690-1694, IEEE, 2014.

[3] L. Boehm, S. Pledl, F. Boegelsack, M. Hitzler, and C. Waldschmidt, "Robotically Controlled Directivity and Gain Measurements of Integrated Antennas at $280 \mathrm{GHz}$," in European Microwave Conference (EuMC), Paris, France, Sep 2015.

[4] M. Hitzler, S. Bader, and C. Waldschmidt, "Key aspects of robot based antenna measurements at millimeter wave frequencies," in Antennas and Propagation (EuCAP), 8th European Conference on, The Hague, Netherlands, pp. 392-396, April 2014.

[5] L. Le Coq, B. Fuchs, T. Kozan, S. Burgos, and P. Iversen, "IETR Millimeter-wave Compact Antenna Test Range Implementation and Validation," in Antennas and Propagation (EuCAP), 2015 9th European Conference on, Lisbon, Portugal, pp. 1-5, April 2015

[6] J. Migl, J. Habersack, C. Hoetzel, A. Geise, C. Schmidt, and H.-J. Steiner, "Performance of Scanned Quiet Zones in Compensated Compact Ranges for Antenna and Payload Testing," in Antenna Measurement Techniques Association (AMTA), Bellevue, USA, AMTA, 2015.

[7] NIST/SEMATECH, "e-Handbook of Statistical Methods." http://itl.nist. gov/div898/handbook/mpc/mpc.htm Accessed: 2015-10-02. 